\title{
UN CAS DE LADRERIE RÉVÉLÉ PAR LES RAYONS $X$
}

\author{
Par STREIGNART
}

L'utilisation des rayons $\mathrm{X}$ en médecine a permis de mettre en évidence bien des formations pathologiques dues à des parasites. Les observations contrôlées de kystes hydatiques siégeant dans différents viscères et dans les os ne sont plus rares. Nous avons eu la bonne fortune d'observer, à la radiographie et tout à fait par hasard, des cysticerques enkystés et calcifiés.

X..., garde-champêtre et accessoirement cultivateur de quelques lopins de terre dans la Hesbaye flamande, nous fut amené pour fracture du tiers inférieur du tibia à la suite d'un accident d'automobile. En dehors de la lésion qui avait motivé l'examen, nous ne fûmes pas peu surpris de trouver, se projetant au niveau de la jambe, et surţout dans sa moitié supérieure, toute une série de calcifications, disposées généralement selon le grand axe du membre et se projetant irrégulièrement sur la silhouette de celui-ci.

Pour avoir vu une image semblable et avoir pris connaissance du texte explicatif donné par l'auteur qui l'a publiée, nous n'avons pas hésité un moment à poser le diagnostic de kystes à cysticerques calcifiés.

En effet, notre image radiographique peut se substituer à celle que Kölher donne dans son bel ouvrage : Roentgenologie - Les limites de l'image normale et pathologique. La description de son unique cas, vérifié par la biopsie, suffit entièrement à commenter la nôtre :

《 Nous avons observé une fois, écrit Kölher, dans l'ombre des muscles du mollet et dans celle du creux poplité, d'autres ombres de densité calcaire ; la figure 108 en représente la forme, la disposition et le nombre.

«Les ombres en question étaient homogènes, larges de 2 à 4 millimètres, et longues de 7 à 12 millimètres; leur axe longitudinal correspondait à peu près à la direction des fibres musculaires; la biopsie confirme le diagnostic que nous avions fait de parasites calcifiés: il s'agissait en effet de cysticerques (C. cellulosæ). (Les ombres de trichines sont généralement rondes et n'ont qu'un ou deux millimètres de diamètre !)

Annales de Parasitologie, T. XI, $\mathrm{N}^{\circ} 1 .-1^{\mathrm{er}}$ janvier 1933 , p. 17-19.

2. 
« Les indications données, ainsi que le nombre élevé de grains de ladrerie, permettent certainement d'éviter un diagnostic erroné.

«Seul, un grain de ladrerie absolument isolé pourrait justifier un diagnostic différentiel avec un ostéome, une petite tumeur cutanée calcifiée, ou un granulome sous-cutané calcifié.

« Un malade, porteur de cysticerques calcifiés, ne souffre pas nécessairement de ténia : cette coïncidence a même été formellement contes-
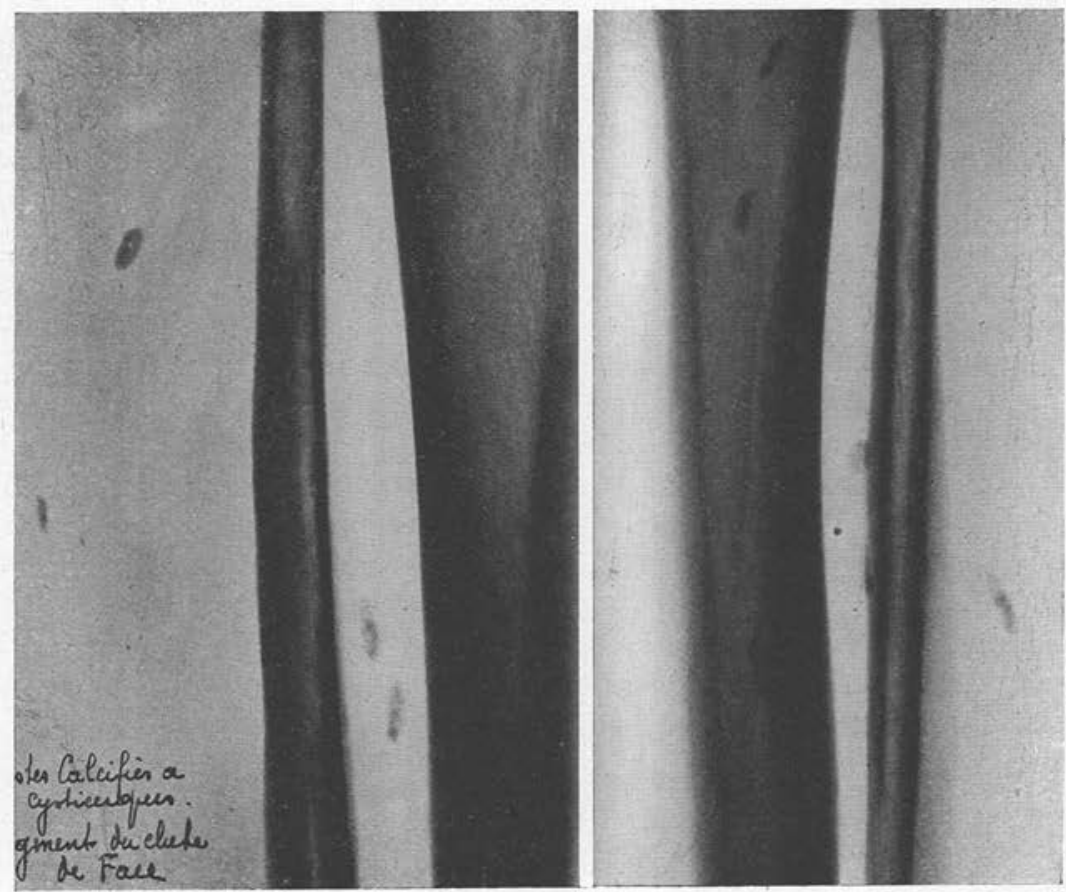

FIg. - Kystes calcifiés à cysticerques. A gauche, fragment du cliché de face ; à droite, fragment du cliché de profil.

tée (par exemple Virchow). Cependant on a décrit, ces derniers temps, un nombre suflisant de cas où l'on a observé la présence simultanée des deux formes (grains et ver adulte chez le même sujet). »

Nous avons demandé au chirurgien qui traite ce cas, de nous prélever une de ces petites calcifications en vue de biopsie, mais le malade s'y est refusé.

Le fait que ce patient pourrait être porteur de cysticerques sans héberger le ténia s'explique aussi par les conditions sociales du blessé. 
Petit fonctionnaire communal, cet homme possède un petit lopin de terre ou un jardin, dans lequel il cultive les légumes nécessaires à la consommation de son ménage. Comme le seul animal domestique qu'il possède - en dehors de la volaille de sa basse-cour - est le cochon qu'il engraisse pour son propre usage et comme le fumier qu'il recueille ainsi est en quantité insuffisante, il se sert aussi de fumier humain. Celui-ci peut contenir des œufs de ténia, qui, ingérés avec les salades ou légumes qu'il mange crus, donnent encore naissance aux cysticerques.

Les images que nous donnons, sont, d'après leurs dimensions, celles de cysticerques arrivés à leur évolution complète et envahis par des sels calcaires, comme le démontre leur densité. Ces cysticerques donnent peu ou pas de signes cliniques dans les localisations sur le tissu conjonctif interfasciculaire des muscles striés. Nous avons cependant interrogé le patient : il dit n'avoir jamais eu la moindre manifestation pathologique et n'avoir jamais expulsé de proglottis. L'examen clinique ne révèle rien. Le blessé refuse, du reste, de nous fournir les matières nécessaires à un examen parasitologique. Toutefois, la description de l'image radiologique de la cysticercose calcifiée, par une autorité en matière de radiodiagnostic telle qu'Alban Kölher, comme, du reste, le diagnostic différentiel avec d'autres lésions qui pourraient créer une image ressemblant à celle que nous avons découverte, nous permettent de conclure qu'il s'agit ici de cysticerques calcifiés.

Le milieu social et le genre de vie du petit cultivateur hesbignon fournissent des arguments indirects. Ces paysans se nourrissent presque exclusivement de viande de porc (saucisses séchées, etc.) et se trouvent dans des conditions excellentes pour s'infecter de ténia. Ajoutons, à cela, que les bêtes sont abattues au domicile du propriétaire et rarement soumises à l'examen du vétérinaire. Ces considérations valent évidemment seulement dans le cas où le patient serait porteur de ténia et de cysticerques en même temps. Il est très probable que notre patient n'a jamais ressenti le moindre trouble aux membres inférieurs.

Il y a peu de cas connus d'images de ce genre. Il est évident que ces cysticerques ne peuvent être mis en évidence que quand ils sont calcifiés et que leur découverte au niveau des membres est due, la plupart du temps, au hasard.

En terminant, je remercie M. le prof. E. Malvoz qui a bien voulu me documenter pour la partie parasitologique, dans l'observation de cette curiosité radiographique. 